\title{
Communications
}

\section{A Monofilar Spiral Antenna Excited Through a Helical Wire}

Hisamatsu Nakano, Yosuke Okabe, Hiroaki Mimaki, and Junji Yamauchi

\begin{abstract}
The radiation characteristics of a monofilar spiral antenna excited through a helical wire are investigated using the method of moments. The spiral has a circumference of more than two wavelengths $\left(2.3 \lambda_{6}\right.$, where $\lambda_{6}$ is the wavelength at a test frequency of $6 \mathrm{GHz}$ ) to obtain a tilted beam. An input impedance of approximately $50 \Omega$ is realized by appropriately selecting the diameter and pitch angle of the helical wire. The antenna shows a gain of approximately $8 \mathrm{~dB}$ relative to an isotropic source in the maximum beam direction $\left(18^{\circ}\right.$ from the direction normal to the antenna plane) and a VSWR frequency bandwidth of approximately $12 \%$.
\end{abstract}

Index Terms-Helical wire, low cross-polarization, spiral antenna, tilted beam formation.

\section{INTRODUCTION}

Conventionally, a monofilar spiral antenna is excited through a straight wire (SW) between the spiral and feed point, as shown in [1] or the inset of Fig. 5, where the spiral is located above a conducting plane to which a coaxial feed line is attached. The spiral antenna having such a straight wire is referred to as an SW-spiral antenna in this paper. The SW-spiral can radiate a tilted beam, which is used for LAN, satellite, and other communication applications.

A problem with the SW-spiral is that a purely resistive input impedance, which is required for impedance matching to a coaxial line feed, is not easily obtained. A solution to this problem is to replace the straight wire with a differently shaped wire, therefore, a helical wire (HX) is used, as shown in Fig. 1(a). The antenna shown in Fig. 1(a) is referred to as an HX-spiral antenna.

This paper investigates the behavior of the input impedance of the HX-spiral, varying the parameters of the helical wire. In addition, the frequency responses of the beam tilt angle, axial ratio, and gain are calculated and discussed, together with the frequency response of the VSWR.

\section{CONFIGURATION}

The HX-spiral antenna to be analyzed is composed of a spiral and a helical wire, as shown in Fig. 1(a). The spiral is Archimedean, as shown in Fig. 1(b): the radius from the center point in the spiral plane to a point on the spiral arm is defined as $r=a_{\mathrm{sp}} \phi$, where $a_{\mathrm{sp}}$ is the spiral constant, and $\phi$ is the winding angle, ranging from $\phi_{\mathrm{sp}}^{s}$ to $\phi_{\mathrm{sp}}^{e}$. The spiral is fixed at a height of $h=\lambda_{6} / 4$ above a conducting plane (which enhances the radiation in the $+z$ hemisphere), where $\lambda_{6}=5 \mathrm{~cm}$ is the wavelength at a test frequency of $6 \mathrm{GHz} \equiv f_{6}$. To obtain a tilted beam, the outer circumference of the spiral, which is approximated as $c_{\mathrm{sp}}=2 \pi a_{\mathrm{sp}} \phi_{\mathrm{sp}}^{e}$, must be more than two wavelengths [1], [2]. In this paper, a circumference of $c_{s p}=11.5 \mathrm{~cm}=2.3 \lambda_{6}$ is used as a test value, generated by a spiral constant of $a_{\mathrm{sp}}=0.0153 \lambda_{6} / \mathrm{rad}$ and an ending angle of $\phi_{\mathrm{sp}}^{e}=23.93 \mathrm{rad}$.

Manuscript received November 7, 2001; revised February 5, 2002

The authors are with the Faculty of Engineering, Hosei University, Koganei, Tokyo 184-8584, Japan (e-mail: nakano@k.hosei.ac.jp).

Digital Object Identifier 10.1109/TAP.2003.809865

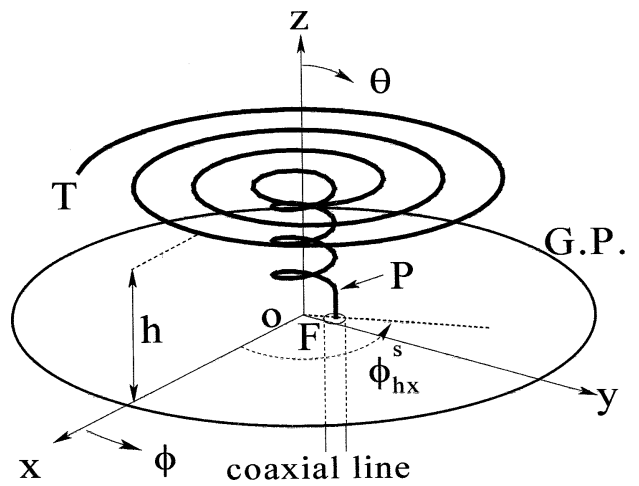

(a)

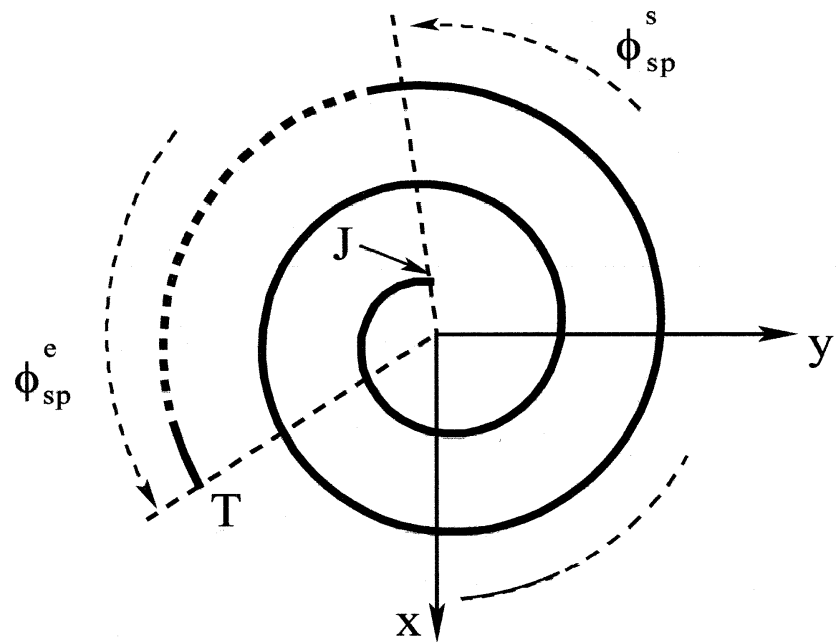

(b)

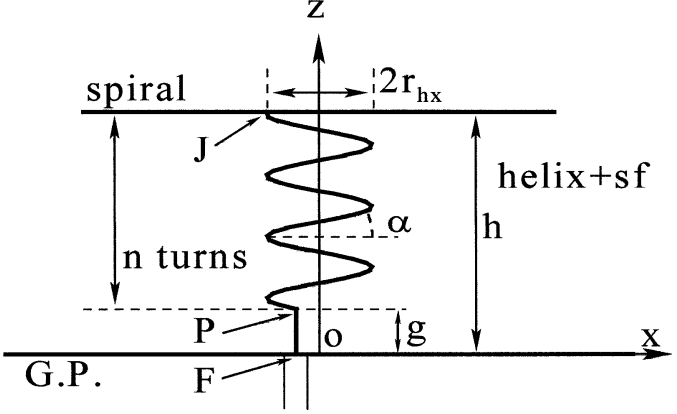

(c)

Fig. 1. Configuration. (a) Spiral antenna excited through a helical wire, designated as an HX-spiral antenna. (b) Spiral wire. (c) Cross-sectional view of the HX-spiral.

The helical wire is specified by the cylinder diameter $2 r_{\mathrm{hx}}$, pitch angle $\alpha$, and number of turns $n$. A short straight filament of length $g$ is inserted between the end of the helical wire $P$ and the feed point $F$, as shown in Fig. 1(a) and (c). This vertically inserted filament facilitates connection of the helical wire to a coaxial line feed. The basic equations for the helical wire are

$$
\begin{aligned}
2 r_{\mathrm{hx}} & =2 a_{\mathrm{sp}} \phi_{\mathrm{sp}}^{s} \\
n & =(h-g) /\left(2 \pi r_{\mathrm{hx}} \tan \alpha\right) \\
\phi_{\mathrm{hx}}^{s} & =\phi_{\mathrm{sp}}^{s}-2 \pi n
\end{aligned}
$$




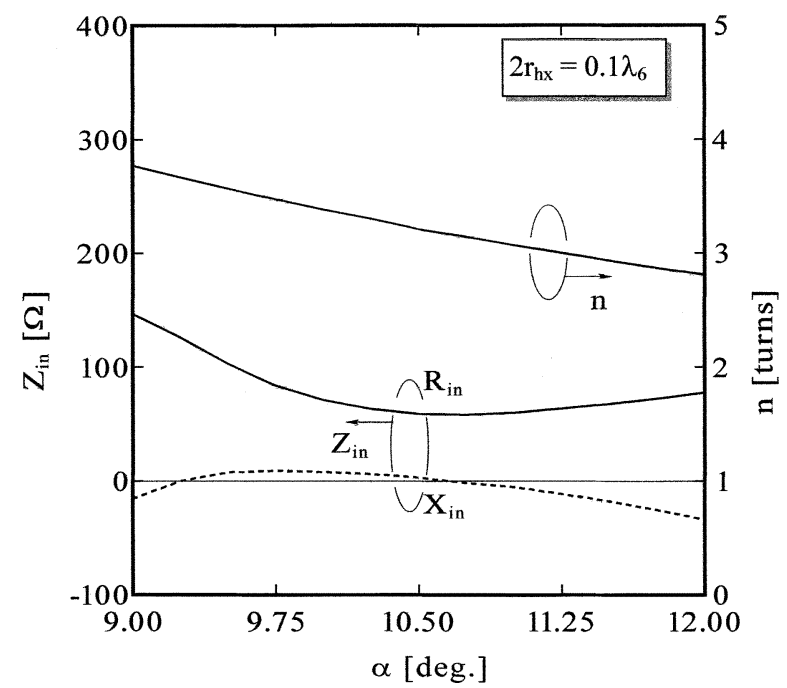

Fig. 2. Input impedance as a function of pitch angle $\alpha$ at a test frequency of 6 $\mathrm{GHz}$.

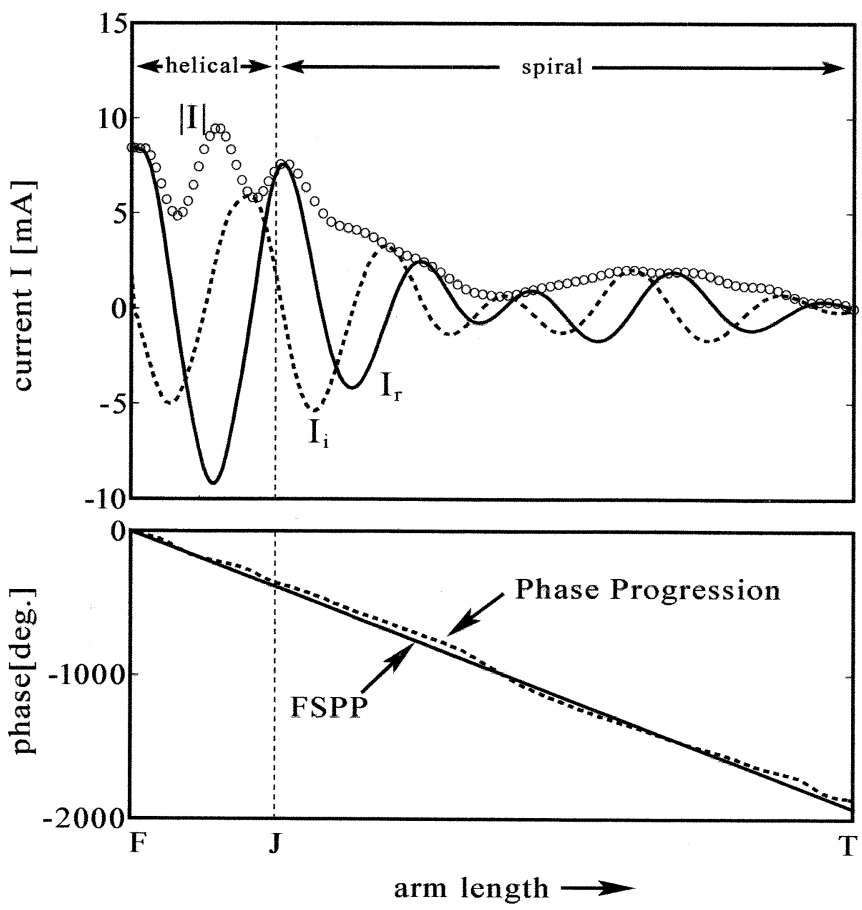

Fig. 3. Current distribution at a test frequency of $6 \mathrm{GHz} ; 2 r_{h x}=0.1 \lambda_{6}$ and $\alpha=10.5^{\circ}$.

where $\phi_{\mathrm{hx}}^{s}$ is the starting angle of the helix. The coordinates of feed point $F$ are given as

$$
x=r_{\mathrm{hx}} \cos \phi_{\mathrm{hx}}^{s} \quad \text { and } \quad y=r_{\mathrm{hx}} \sin \phi_{\mathrm{hx}}^{s} .
$$

The helix diameter $2 r_{\mathrm{hx}}$ and pitch angle $\alpha$ are varied for investigating the VSWR and tilted beam formation. Note that varying the helix diameter $2 r_{\mathrm{hx}}$ changes the starting angle $\phi_{\mathrm{sp}}^{s}$ of the spiral, as seen from (1). Also note that varying $\alpha$ changes both the number of turns $\mathrm{n}$ and the starting angle of the helix $\phi_{\mathrm{hx}}^{s}$ (and correspondingly, the coordinates of feed point $F$ ), as seen from (2) and (3).

The conducting plane below the spiral is assumed to be of infinite extent in the numerical analysis. In the experiment, a large square conducting plane, with a side length $L_{s}$ of approximately nine wavelengths, is used to approximate the infinite conducting plane. Note that a preliminary theoretical investigation shows that when the
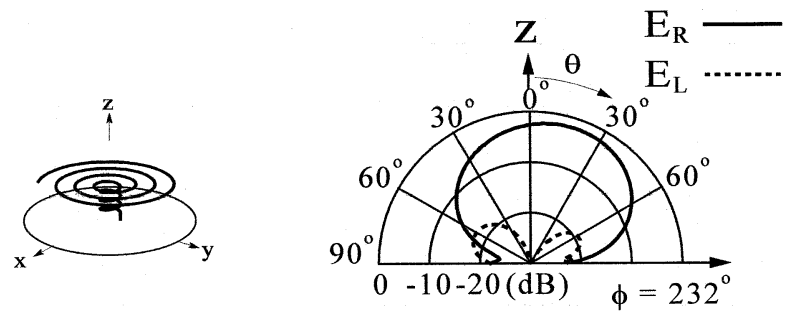

(a)
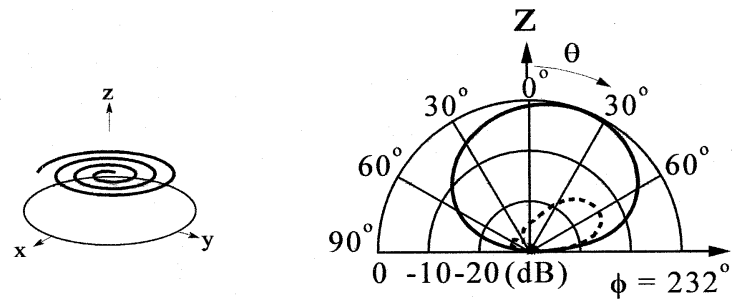

(b)

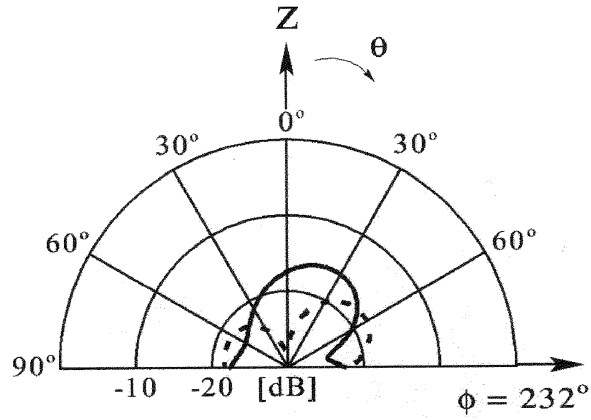

(c)

Fig. 4. Maximum beam cut in the $z-\phi$ plane $\left(\phi=232^{\circ}\right)$ for an HX-spiral antenna with $2 r_{h x}=0.1 \lambda_{6}$ and $\alpha=10.5^{\circ}$ at a test frequency of $6 \mathrm{GHz}$. (a) Total radiation pattern. (b) Partial radiation from the spiral. (c) Partial radiation from a vertical section composed of a helical wire and a short straight filament (helix+sf)

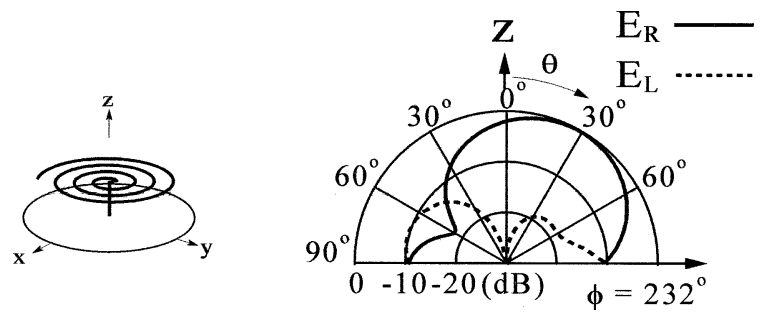

Fig. 5. Maximum beam cut in the $z-\phi$ plane $\left(\phi=232^{\circ}\right)$ for an SW-spiral antenna at a test frequency of $6 \mathrm{GHz}$.

side length $L_{s}$ is greater than three wavelengths, the input impedance $Z_{\text {in }}$ (and hence the VSWR) is close to that obtained with a conducting plane of infinite extent.

\section{NUMERICAL RESUlTS AND DISCUSSION}

Analysis of the HX-spiral is performed using the method of moments (MoM) [3], where the wire radii of the spiral and helical wires are chosen to be very small: $\rho \ll \lambda_{6}\left(\rho=0.008 \lambda_{6}\right.$ is used).

The input impedance $Z_{\text {in }}=R_{\text {in }}+j X_{\text {in }}$ for a helix diameter ranging from $2 r_{\mathrm{hx}}=0.08 \lambda_{6}$ to $0.118 \lambda_{6}$ is investigated at a test frequency of $f_{6}$. Fig. 2 shows an example of $Z_{\text {in }}$ for $2 r_{\mathrm{hx}}=0.1 \lambda_{6}$, where a short straight filament length of $g=0.0625 \lambda_{6}$ is used. For reference, the number of helical turns $n$ as a function of the pitch angle $\alpha$, calculated from (2), is also illustrated in this figure. It is found that 


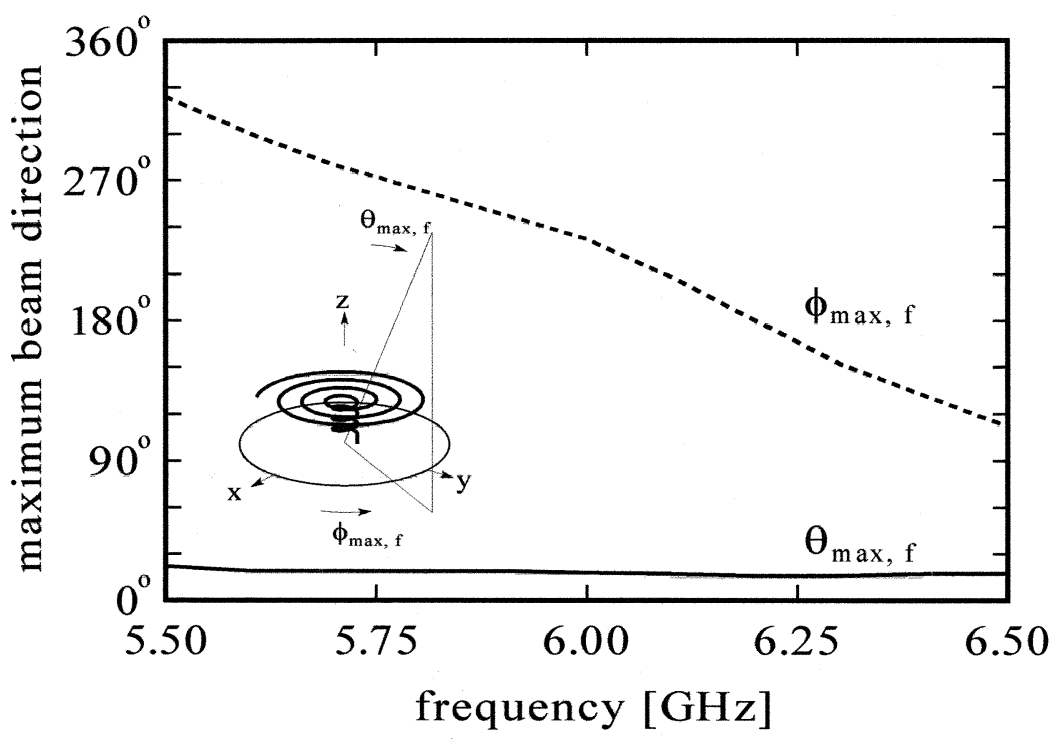

Fig. 6. Beam tilt angle, $\theta_{\max , f}$ and $\phi_{\max , f}$, as a function of frequency for an HX-spiral with $2 r_{h x}=0.1 \lambda_{6}$ and $\alpha=10.5^{\circ}$.

an input impedance of approximately $50 \Omega$, which can be matched to commercially available coaxial lines, is obtained at $\alpha=10.5^{\circ}$. This HX-spiral with $2 r_{\mathrm{hx}}=0.1 \lambda_{6}$ and $\alpha=10.5^{\circ}$ is referred to as an $\mathrm{HX}_{50}$-spiral throughout. Note that the corresponding $\mathrm{SW}$-spiral has an input impedance of $209-j 31 \Omega$.

Fig. 3 shows the current distribution of the $\mathrm{HX}_{50}$-spiral at a test frequency of $f_{6}$, where the phase progression (dotted line) is presented together with the free-space phase progression (FSPP, solid line). As observed from this figure, the current smoothly flows along the spiral as a traveling wave. Fig. 4(a) shows the beam cut in the maximum radiation plane ( $z-\phi$ plane with $\phi=232^{\circ}$ ) of the $\mathrm{HX}_{50}$-spiral at $f_{6}$, where $E_{R}$ (solid line) shows a right-hand circularly polarized wave component (copolarization component) and $E_{L}$ (dotted line) shows a left-hand circularly polarized wave component (cross-polarization component). For comparison, the beam cut in the maximum radiation plane for the corresponding SW-spiral is shown in Fig. 5. It is observed that the cross-polarization component for the $\mathrm{HX}_{50}$-spiral is smaller than that for the $\mathrm{SW}$-spiral, as desired. The smaller cross-polarization component for the $\mathrm{HX}_{50}$-spiral is attributed to the fact that the contribution made by the helical wire and short filament [this vertical section is designated as helix + sf, as shown in Fig. 1(c)] to the total radiation is lower than that made by the vertical straight wire for the SW-spiral. Note that Fig. 4(b) and (c) are the radiation patterns evaluated from the partial currents along the spiral and helix + sf, respectively, derived from the total current distribution shown in Fig. 3.

The tilted beams of the $\mathrm{HX}_{50}$-spiral at frequencies lower and higher than $f_{6}$ (at 5.6 and $6.4 \mathrm{GHz}$, not shown in this paper) are also compared with those of the SW-spiral antenna. The comparison reveals that, as in the comparison at $f_{6}$ [Figs. 4(a) and 5], the $\mathrm{HX}_{50}$-spiral has an advantage over the SW-spiral in that the tilted beam has a reduced cross-polarization component.

Fig. 6 shows the beam tilt angle (maximum beam direction) for the $\mathrm{HX}_{50}$-spiral as a function of frequency $f$, which is specified by $\theta=\theta_{\max , f}$ and $\phi=\phi_{\max , f}$. It is revealed that the angle measured from the $z$ axis, $\theta_{\max , f}$, remains almost unchanged, while the angle measured from the $x$ axis, $\phi_{\max , f}$, varies in a nearly linear fashion as a function of frequency. This behavior is commonly found in monofilar spiral antennas [1].

Fig. 7 shows the frequency response of the $\mathrm{HX}_{50}$-spiral, where the axial ratio $A R$ and gain $G_{R}$ (for a right-hand circularly polarized wave) are evaluated in the maximum beam direction observed at frequency

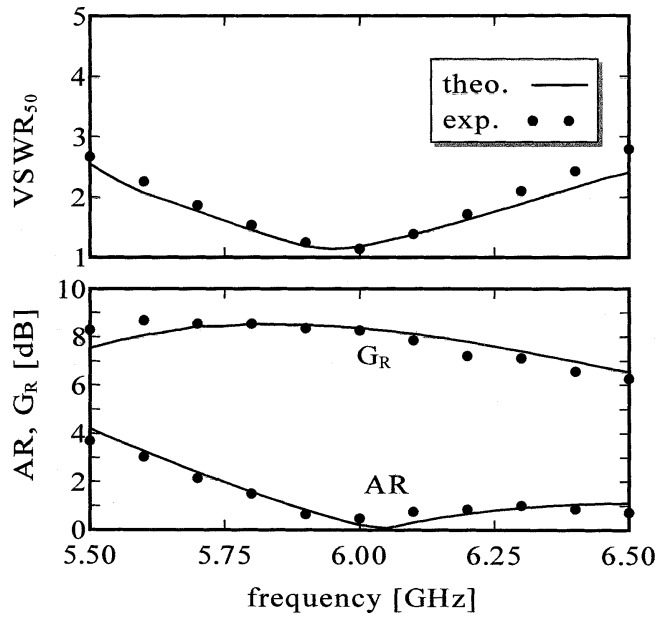

Fig. 7. VSWR relative to a 50-ohm impedance, axial ratio $A R$, and gain $G_{R}$ as a function of frequency for an HX-spiral with $2 r_{h x}=0.1 \lambda_{6}$ and $\alpha=10.5^{\circ}$.

$f_{6}\left(\theta=18^{\circ} \equiv \theta_{\max , 6}\right.$ and $\left.\phi=232^{\circ} \equiv \phi_{\max , 6}\right)$. The frequency bandwidth for a VSWR $=2$ criterion is calculated to be approximately $12 \%$. Within this bandwidth, the $\mathrm{HX}_{50}$-spiral radiates a circularly polarized wave with an axial ratio of less than $3 \mathrm{~dB}$. Note that the gain $G_{R}$ at $f_{6}$ is $8.4 \mathrm{~dB}$ relative to an isotropic source and its variation within the above-mentioned $12 \%$ bandwidth is small (approximately $1 \mathrm{~dB}$ ), as desired.

\section{CONCLUSION}

A monofilar spiral antenna excited through a helical wire (HX-spiral antenna) can have an input impedance of approximately $50 \Omega$ by appropriately selecting the helix diameter and pitch angle. The cross-polarization component of the radiation is lower than that of a corresponding $\mathrm{SW}$-spiral. The HX-spiral realizes a maximum tilt angle $\theta_{\max , 6}=18^{\circ}$ from the $z$ axis at a test frequency of $6 \mathrm{GHz}$, with a gain of approximately $G_{R}=8.4 \mathrm{~dB}$ relative to an isotropic source. The HX-spiral shows a frequency bandwidth of approximately $12 \%$ for a VSWR $=2$ criterion. Within this bandwidth, the radiation field is circularly polarized with an axial ratio of less than $3 \mathrm{~dB}$, and the gain variation is small (approximately $1 \mathrm{~dB}$ ). 


\section{ACKNOWLEDGMENT}

The authors would like to thank V. Shkawrytko for his kind assistance in the preparation of this manuscript.

\section{REFERENCES}

[1] H. Nakano, Y. Shinma, and J. Yamauchi, "A monofilar spiral antenna and its array above a ground plane-formation of a circularly polarized tilted fan beam," IEEE Trans. Antennas Propagat., vol. AP-45, pp. 1506-1511, Oct. 1997

[2] J. A. Kaiser, "The Archimedean two-wire spiral antenna," IRE Trans Antennas Propagat., vol. AP-8, no. 3, pp. 312-323, May 1960.

[3] H. Nakano, Analysis Methods for Electromagnetic Wave Problems, E. Yamashita, Ed. Boston, MA: Artech House, 1996, vol. II, ch. 3.

\section{An Experimental Study of Antenna Array Calibration}

Inder J. Gupta, James R. Baxter, Steven W. Ellingson, Hyung-Geun Park, Hyun Seo Oh, and Mun Geon Kyeong

\begin{abstract}
The coupling matrix concept for predicting the radiation patterns of elements of an antenna array is studied. Measured data, as well as some numerical data, are used in the study. It is demonstrated that for some practical antennas of interest whose radiation patterns are affected by structure scattering, the coupling matrix concept may not work very well. As expected, the stronger the structure scattering, the greater the discrepancy between the predicted patterns and the actual patterns.
\end{abstract}

Index Terms-Antenna calibration, coupling matrix, structure scattering.

\section{INTRODUCTION}

For angle-of-arrival (AoA) estimation and certain smart antenna processing techniques, one needs to know the array manifold for all incidence angles. This requirement can be relaxed if the array manifold depends entirely on the antenna geometry and radiation pattern of a single antenna in free space. In practice, this is rarely true. The coupling between various elements of an antenna array as well as scattering by the antenna mounting structure and/or other nearby structure affects the array manifold. Also, there will be differences among the cabling and receivers used to down convert and digitize the signals from each antenna element. All of these effects have the potential to vary in both time and frequency, making the array manifold very difficult to anticipate/estimate. The process of obtaining the array manifold is called antenna-array calibration.

Many different algorithms [1]-[5] have been reported in the literature for antenna-array calibration. Most of these algorithms depend on the assumption that the voltages received by an $N$ element antenna array for a signal incident from direction $\phi$ can be written as

$$
\mathbf{b}(\phi)=\mathbf{C Z a}(\phi)
$$

Manuscript received December 21, 2001; revised February 2001. This work was supported in part by the Electronics and Telecommunications Research Institute, Taejon, South Korea.

I. j. Gupta, J. R. Baxter, and S. W. Ellingson are with the The Ohio State University ElectroScience Laboratory, Department of Electrical Engineering, Columbus, OH 43212 USA.

H. G. Park, H. S. Oh, and M. G. Kyeong are with Electronics and Telecommunications Research Institute, (ETRI) Daejon, Korea.

Digital Object Identifier 10.1109/TAP.2003.809870 where $\mathbf{b}$ is an $N \times 1$ vector of the measured voltages, $\mathbf{C}$ is an $N \times N$ matrix which represents variations in the complex gain among antennas, feedlines, and receivers, $\mathbf{Z}$ is an $\mathbf{N} \times \mathbf{N}$ matrix representing the effect of antenna-to-antenna coupling and structure scattering, and $\mathbf{a}(\phi)$ is an $N \times 1$ vector describing the received voltages in the absence of antenna-to-antenna coupling and structure scattering. Note that $\mathbf{a}(\phi)$ depends only on the antenna geometry and the radiation pattern of a single antenna element. Let us say that we are interested in the antenna effects only. In that case, matrix $\mathbf{C}$ can be replaced with an identity matrix and (1) can be written as

$$
\mathbf{b}(\phi)=\mathbf{Z a}(\phi) .
$$

Assuming that the antenna geometry is known, then according to (2), one needs to know only $\mathbf{Z}$ to predict the antenna array manifold for any $\phi$. The question is how to estimate $\mathbf{Z}$ ? Various algorithms to estimate $\mathbf{Z}$ are given in the references cited. The simplest of these assumes that the vector $\mathbf{b}(\phi)$ is measured for $M$ independent (widely separated and not coincident with grating lobes), directions such that $M \geq N$. Let these measurements be represented by the $N \times M$ matrix $\mathbf{B}_{M}=\left[\mathbf{b}_{1} \mathbf{b}_{2} \cdots \mathbf{b}_{M}\right]$. Then (2) can be rewritten as [3]

$$
\mathbf{B}_{M}=\mathbf{Z} \mathbf{A}_{M}
$$

where $\mathbf{A}_{M}=\left[\mathbf{a}_{1} \mathbf{a}_{2} \cdots \mathbf{a}_{M}\right]$. (3) is an overdetermined set of linear simultaneous equations. The least square solution for $\mathbf{Z}$ is given by

$$
\mathbf{Z}=\mathbf{B}_{M} \mathbf{A}_{M}^{H}\left(\mathbf{A}_{M} \mathbf{A}_{M}^{H}\right)^{-1} .
$$

Next, one can use this $\mathbf{Z}$ to predict $\mathbf{b}(\phi)$ for any $\phi$. However, (2), in general, does not have a way to deal with structure scattering because it (structure scattering) cannot be described in a few parameters. For large antenna arrays, where structure scattering affects all the antenna elements in a similar fashion, one may be able to find an appropriate $Z$, provided that the embedded element pattern includes structure scattering effects. For small antenna array the same may not be true. To test this hypothesis, we built two experimental antenna arrays. The radiation patterns of these antenna arrays were measured in The Ohio State University compact range [6]. The measured patterns for a few directions were then used to estimate matrix $\mathbf{Z}$. Next, matrix $\mathbf{Z}$ is used to predict $\mathbf{b}$ for all angles. In this communication, the predicted results are compared with the measured results. It is shown that for the two antenna arrays, (2) does not work very well. The same is demonstrated using numerical results [method of moments (MoM) solution] for a simple antenna array.

The rest of the communication is organized as follows: The experimental antenna arrays and their measured and estimated patterns are discussed in Section II. Section III contains the numerical results from the simulated array. Finally, Section IV contains a summary and the general conclusions of this work.

\section{EXPERIMENTAL ANTENNA ARRAYS}

The experimental antenna arrays are linear arrays of eight elements each and are designed to operate around $2.45 \mathrm{GHz}$. One of the antenna arrays has small interelement spacing $(\sim 0.4 \lambda)$, whereas, the other antenna element has large interelement spacing $(\sim 0.75 \lambda)$. The details of these antennas and their measured and predicted patterns are discussed in this section.

\section{A. Antenna Array \#1}

The first antenna array, shown in Fig. 1, consists of eight printed dipoles. Each printed dipole is fed by a coaxial cable through a $\lambda / 4$ 